\title{
Status of the KTH-NASA Wind-Tunnel Test for Acquisition of Transonic Nonlinear Aeroelastic Data
}

\author{
Walter A. Silva* \\ NASA Langley Research Center, Hampton, Virginia \\ Ulf Ringertz, ${ }^{\dagger}$ Gloria Stenfelt, ${ }^{\ddagger}$ and David Eller ${ }^{\S}$ \\ Kungliga Tekniska Högskolan, or Royal Institute of Technology, SE-100 44 Stockholm, Sweden \\ Donald F. Kellerף and Pawel Chwalowskil \\ NASA Langley Research Center, Hampton, Virginia
}

\begin{abstract}
This paper presents a status report on the collaboration between the Royal Institute of Technology (KTH) in Sweden and the NASA Langley Research Center regarding the design, fabrication, modeling, and testing of a full-span fighter configuration in the Transonic Dynamics Tunnel (TDT). The goal of the test is to acquire transonic limit-cycleoscillation (LCO) data, including accelerations, strains, and unsteady pressures. Finite element models (FEMs) and aerodynamic models are presented and discussed along with results obtained to date.
\end{abstract}

\section{Introduction}

There is significant interest within the aerospace community in the ability to model, analyze, and predict nonlinear aeroelastic limit cycle oscillations (LCOs). Although there is a significant amount of research being performed across several organizations in this area, a major limitation to this research is the availability of high-quality, experimental transonic LCO data [1-12].

This paper presents the status of a collaborative effort between the Royal Institute of Technology (KTH) and the NASA Langley Research Center to acquire a high-quality LCO database on a full-span fighter configuration. The configuration selected will be discussed first, followed by a description of the design, fabrication process, instrumentation, and preliminary data acquired to date.

\section{Wind-Tunnel Model Configuration}

In 1985 and 1986, two wind-tunnel models of the Saab JAS 39 Gripen were designed, built, and tested in the NASA Transonic Dynamics Tunnel (TDT) for flutter clearance. One model, referred to as the stability model, was designed to be stiff but incorporated proper scaling of both the mass and geometry. The other model, referred to as the flutter model, was also designed for proper scaling of structural dynamics and was used for flutter testing with various external stores attached.

For the current collaboration, a single generic fighter flutter-model version of these earlier models was selected. The new model, shown in Figure 1, has a similar outer mold line (OML) to the Gripen, but it has been modified to provide a more generic fighter configuration. Specifically, the air intakes were removed from the fuselage, and the wing received an aspect ratio increase and a leading-edge sweep reduction.

\footnotetext{
*Senior Research Scientist, Aeroelasticity Branch, AIAA Associate Fellow.

${ }^{\dagger}$ Professor, Department of Aeronautical and Vehicle Engineering, AIAA Senior Member.

$¥$ Graduate Reseacher, Department of Aeronautical and Vehicle Engineering, AIAA Senior Member.

$\S$ Professor, Department of Aeronautical and Vehicle Engineering, AIAA Senior Member.

ף Aerospace Engineer, Aeroelasticity Branch.

" Aerospace Engineer, Aeroelasticity Branch.
} 


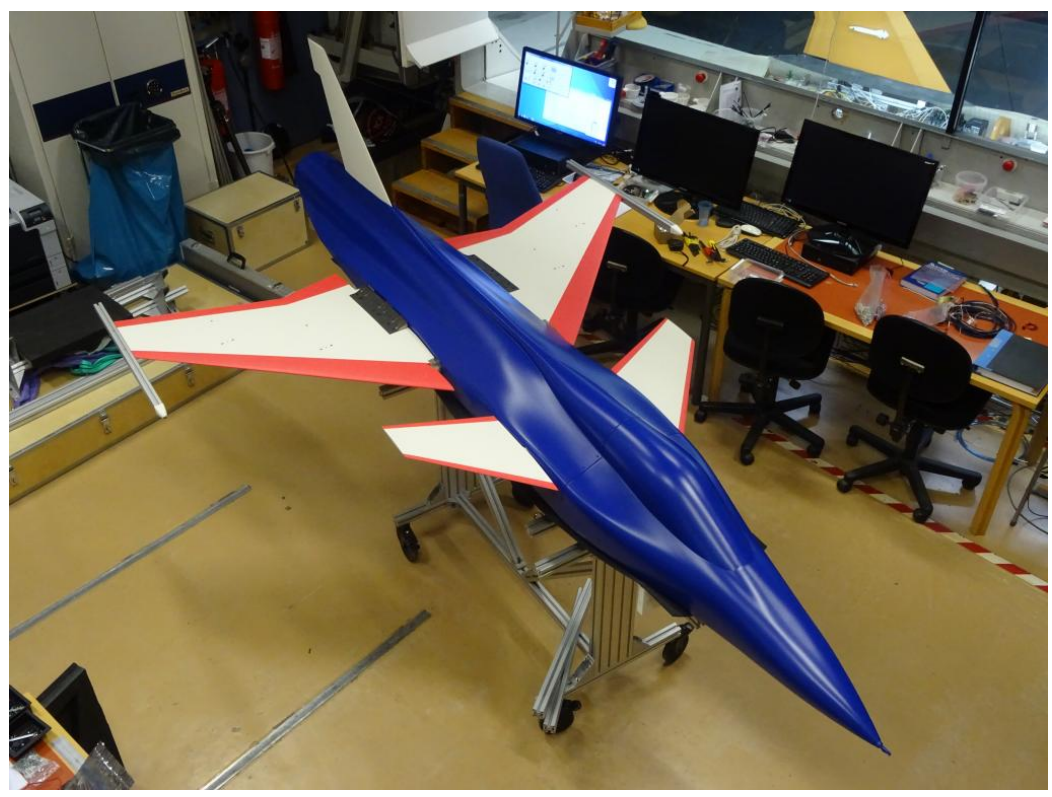

Figure 1: The generic fighter aeroelastic wind-tunnel model to be tested in 2016.

\section{Design and Fabrication Process}

The design of the new fuselage and wings was performed using modern computational tools. Initially, the main aerodynamic surfaces were sketched using the aircraft design and mesh tool SUMO [13]. Preliminary aerodynamics were then computed via the automatic creation of an unstructured mesh and subsequent calculations performed with the potential flow solver DWFS [14].

The new wing design is based on the project 2111 [15] planform which was a candidate for the JAS 39 Gripen but was not selected for the final design of that aircraft. All wing sections have the NACA65A005 airfoil which is a symmetric airfoil suitable for transonic flight conditions. The wing was designed for attached flow with the goal of improving the sustained turn rate of the aircraft but with reduced high speed performance. The Computational Fluid Dynamics (CFD) software EDGE [16] was used to investigate the aerodynamic properties of the modified overall design. The new fuselage shell for the generic fighter model was modified to remove the air intakes and then fit to the internal load carrying structure, which is a single spar that is not structural-dynamically scaled to any specific configuration.

For fabrication purposes, the aerodynamic surface geometry was imported into Unigraphics NX 8, a Computer Aided Design (CAD) tool. The fuselage geometry was straightforward and was imported directly from SUMO. The wing required an intermediate step because the SUMO design gave it a sharp traling edge which is impossible from a production point of view. A trailing-edge thickness of at least $1 \mathrm{~mm}$ is required for a wind-tunnel model of this size. Therefore, the trailing-edge gap was adjusted using the airfoil analysis tool XFLR5, where the blending distance from the leading edge was chosen to be $80 \%$. The number of defining points for the airfoil were also refined in XFLR5 to ensure a well-defined spline fit in the CAD tool, considering that the wings were to be manufactured based on the CAD geometry. Further changes to the surface geometry in the CAD environment were only performed in terms of splitting surfaces and trimming, e.g., close to the wing connection to the fuselage.

For the fabrication of the wings and fuselage, molds were prepared in the CAD environment and then milled using a computer numeric controlled (CNC) machine. The molds, shown for the wing in Figure 2, were then used for the hand lay-up of the composite wing and fuselage shells. The material used was a glass fiber reinforced epoxy laminate combination, selected for its high failure strain. Different fiber lay-ups were used for the fuselage and wing. Internal ribs and stringers were made of PVC core material used for sandwich construction. Local reinforcements were made for the wing-to-fuselage interface and also for the pylon attachments. All of these inner construction parts were modeled in CAD, and based on these parts, a preliminary finite element model for structural analysis was generated in NX Nastran. Future work will 
involve tuning and adapting this finite element model to match the properties of the elastic wing, to model its attachment to the load-carrying structure, and to model nonlinear mechanics. For the full configuration, which is to be tested in the TDT, extensive work is expected to be put into tuning the analytical model to correlate with experimental results.

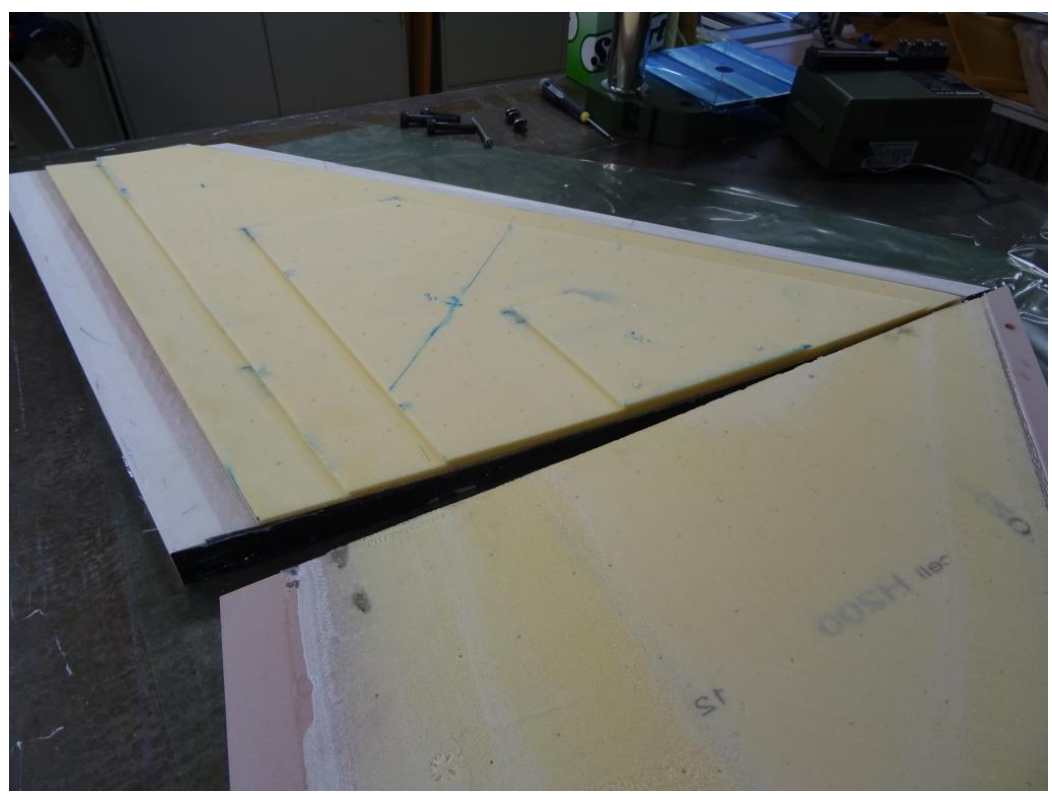

Figure 2: Wing shells during manufacturing in the computer-milled molds.

\section{Instrumentation}

\section{A. Pressure Taps}

Static and dynamic pressure measurements on the surface of a model are key components in a high-quality dataset used for aeroelastic code validations and predictions. Consequently, an important upgrade from the original wind-tunnel model program has been the installation of wing pressure taps, which can be seen in Figure 3. It should be mentioned that the particular method used to install the pressure taps is evolving. Therefore, the discussion that follows is intended to provide the reader with a general sense of the pressure measurement strategy and not necessarily the final design or installation strategy.

An early approach for installing pressure taps was based on a particular design and construction technique developed in a previous project $[17,18]$ at $\mathrm{KTH}$. This technique involves using thin stainless steel tubes mounted flush to the inside of the wing skin. The first step is to drill matching small holes, typically 0.5 $\mathrm{mm}$, through the wing skin and through the wall of a steel tube for each desired installation location. A guide pin is used to align the wing skin hole with the hole in the steel tube, and then the tube is fastened to the wing skin with an epoxy adhesive, as shown in Figure 4.

One end of the tube is firmly affixed to the skin with epoxy resin, and the other end is connected to a flexible plastic tube that leads to a miniature electronic pressure scanner. The process is repeated for each pressure tap, and the entire installation is tested for leaks before the wing skins are joined to form the wing structure. This method for installing the pressure taps is evolving so the final method used for the high-speed wings for the TDT test may differ from this one.

The wing is equipped with 32 taps at two span-wise locations for a total of 64 taps. The inboard location is at the midpoint between the inner and outer pylon, and the outboard location is at the midpoint between the outer pylon and the wing tip.

The use of electronic pressure scanners for unsteady testing, considering phase shifts and time delays, is discussed and analyzed in detail in the references [17,18]. There are still uncertainties in the relative timing of the measurements. Therefore, the plan for the current collaborative effort is to use the scanners 
in combination with additional unsteady piezoresistive pressure sensors with at least one installed at each span location for both the upper and lower surface (minimum total of eight). This type of sensor typically has much better phase accuracy and can, therefore, be used to correct the phase of the scanner pressures, as described in the work by Huebner, Bergmann and Loeser [19].

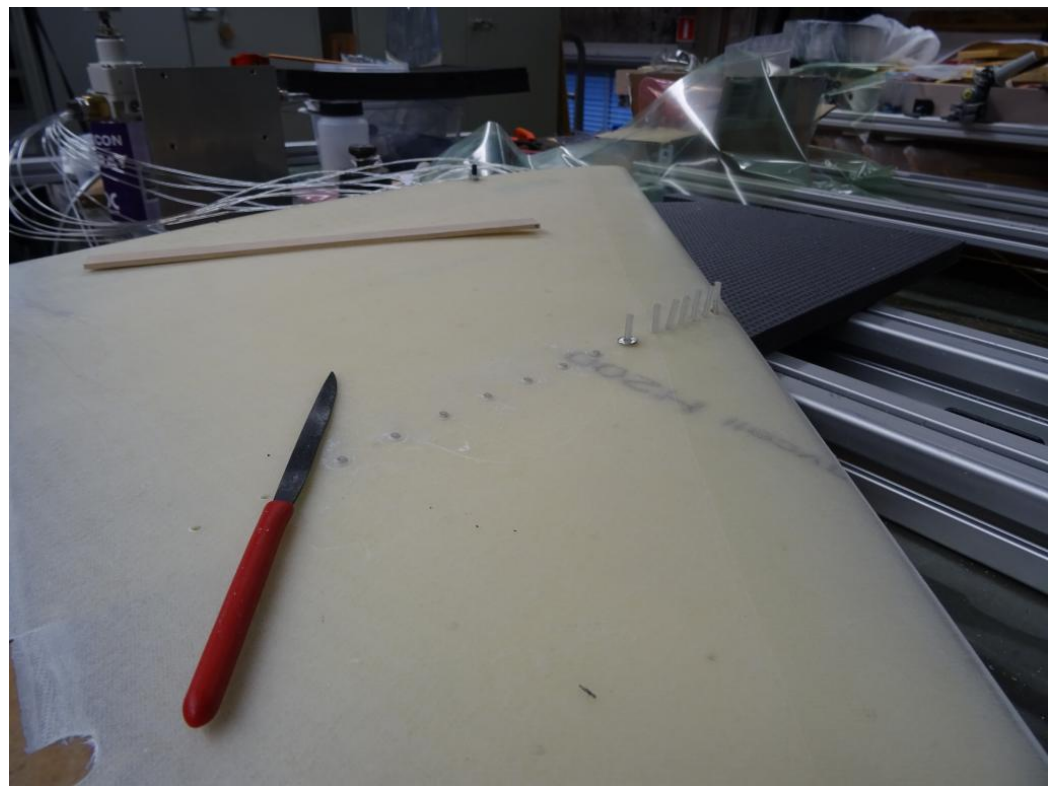

Figure 3: Layout of tubing used for pressure taps in the computer milled molds.

\section{B. Optical Displacement Measurements}

Model surface displacements will be measured by a non-contact optical positioning system called Qualisys [20]. This system of optical cameras and discrete reflective targets has already been used at the lowspeed wind tunnel at KTH for initial testing of the low-speed version of the current model configuration. This system captures the positions of the reflective targets at a rate of 500 to $1000 \mathrm{~Hz}$, depending on the chosen resolution. The system also has the capability to capture high-speed video with the same resolutiondependent frame rate. Synchronizing this system with other sensor output will be part of the future work in this project and is part of the overall development of the data acquisition system. A photo showing the use of this displacement measurement system for stiffness testing is presented as Figure 6. It should be mentioned that the large spherical targets are used for calibration purposes and are not the targets to be used for transonic testing.

\section{Data Acquisition}

A significant issue associated with testing a wind-tunnel model with a large number of sensors is the routing of all the cables to a data acquisition (DAQ) system outside the tunnel test section. For the current model, approximately 100 strain gauge bridges and accelerometers are planned together with 64 pressure taps for unsteady pressure measurements. Each sensor uses 2-4 wires, resulting in several hundred wires carrying sensitive analog signals through fairly long cables, typically at least 30 meters in length, from the sensor to the data acquisition system outside the test section. This type of arrangement requires significant time in the wind tunnel to route all of the wires and cables, verify that each sensor is functioning properly via end-to-end checks, and then secure all components before the testing can begin.

In the present project, the requested test section occupation time in the TDT is limited by funding, so the model installation needs to be efficient. Fortunately, due to rapid development in computer and sensor technology, new hardware solutions have become available in much smaller sizes. There are now data acquisition systems small enough to fit inside the fuselage of the wind-tunnel model and such a system is 


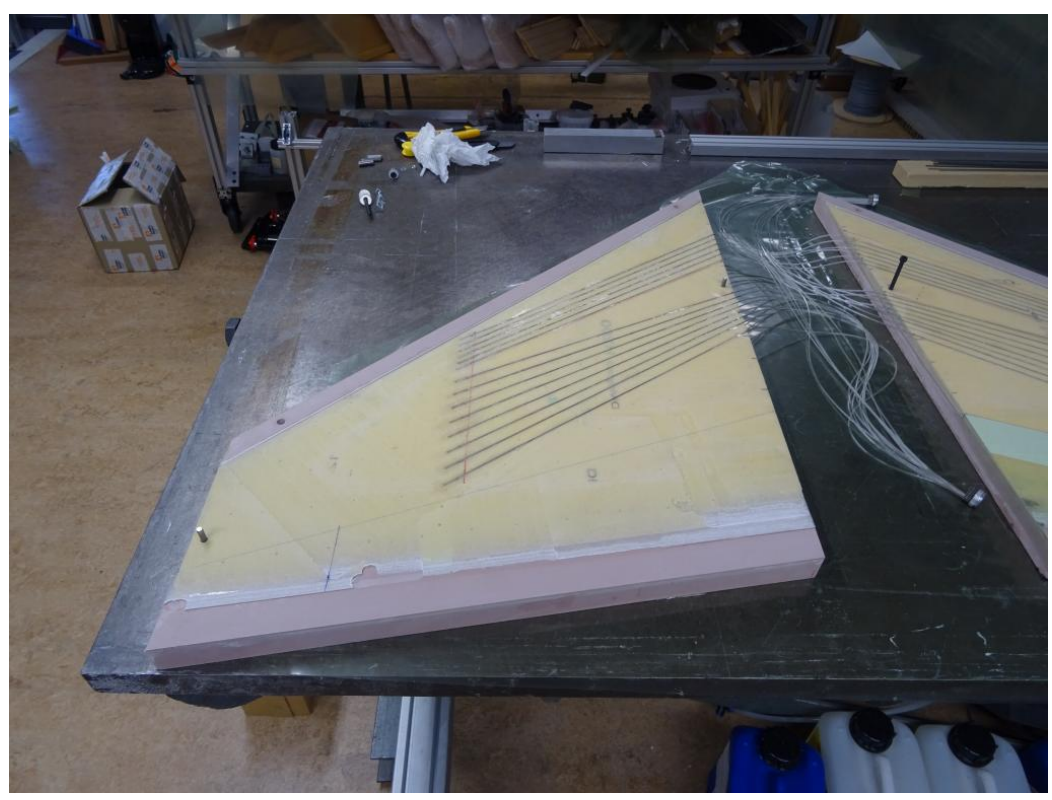

Figure 4: Detailed photo of pressure tap installation.

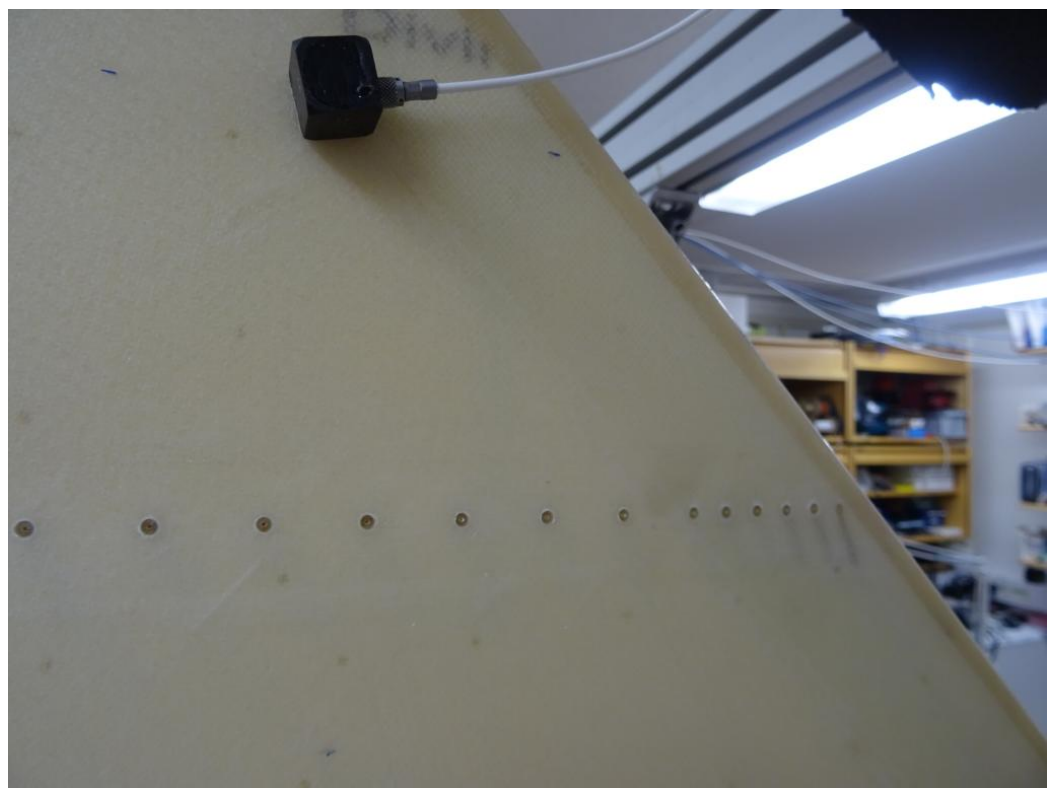

Figure 5: Detailed photo of pressure tap locations on wing. 


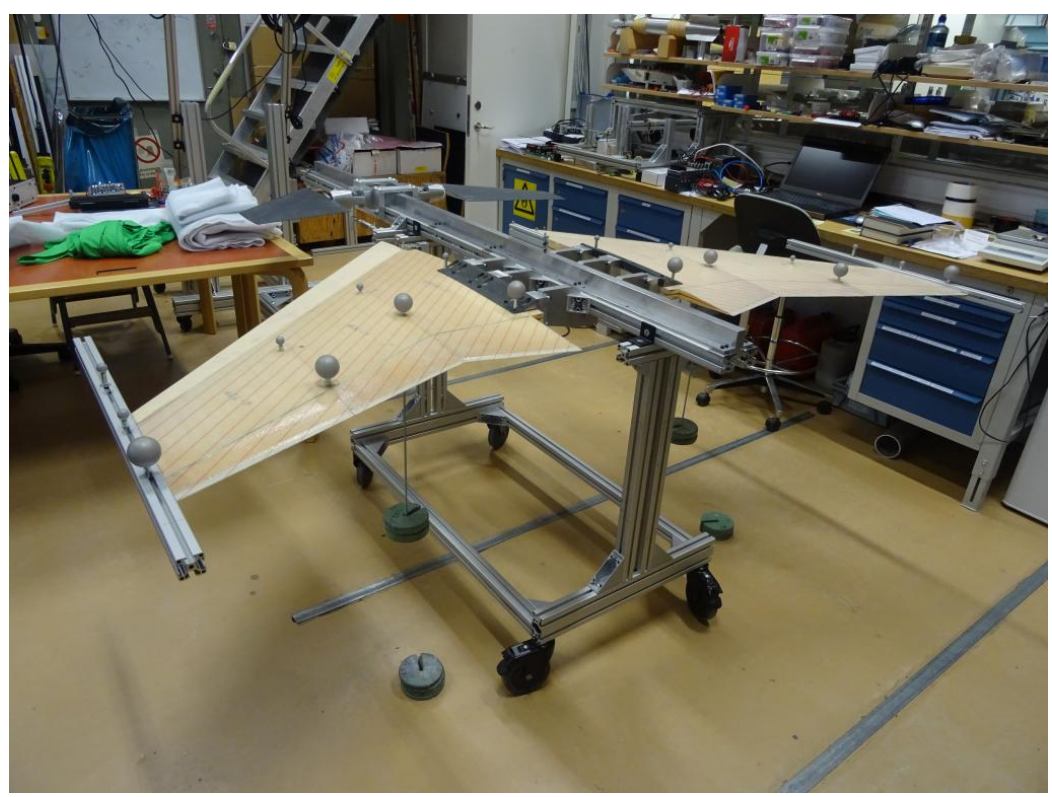

Figure 6: Stiffness test using optical markers and dead weights.

currently in development at KTH, as shown in Figure 7. Moving components of the data acquisition system inside the fuselage would eliminate several of the aforementioned installation steps.

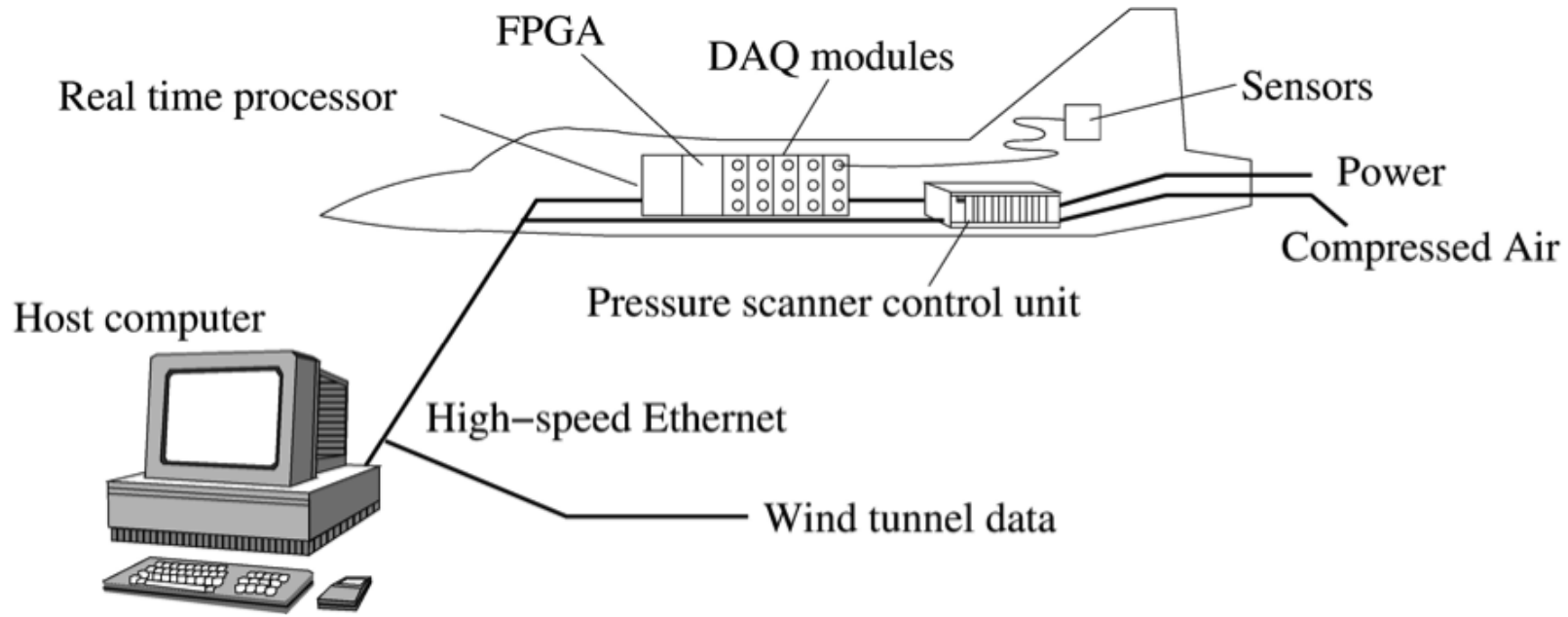

Figure 7: Measurement and control system overview. The FPGA is a field programmable gate array to faciliate data processing.

\section{Transonic Dynamics Tunnel}

Testing of the new generic fighter configuration wind-tunnel model will be conducted in the NASA Langley Transonic Dynamics Tunnel. The TDT is a unique national facility dedicated to identifying, understanding, and solving relevant aeroelastic problems. The TDT is a closed-circuit, continuous-flow, variable-pressure wind tunnel with a 16-foot square test section with cropped corners (Figure 8). The tunnel uses either air or a heavy gas as the test medium and can operate at stagnation pressures from near vacuum to atmospheric, has a Mach number range from near zero to 1.2, and is capable of achieving maximum Reynolds numbers of 
about 3 million per foot in air and 10 million per foot in heavy gas. Prior to 1998, the TDT used dichlorodifluoromethane, R-12, as the aerodynamic test medium. The TDT now uses 1,1,1,2 -tetrafluoroethane, R-134a, as the test medium [21,22]. Testing in heavy gas has important advantages over testing in air: improved model to full-scale similitude (which results in cheaper, heavier models with lower model elastic mode frequencies), higher Reynolds numbers, and reduced tunnel power requirements. The TDT is specially configured for flutter testing, with excellent model visibility from the control room and a rapid tunnel shutdown capability for model safety (bypass valves). Model mount systems include a sidewall turntable for semispan models, a variety of stings for full-span models and a cable-mount system for "flying" models. The TDT also offers an airstream oscillation system for gust studies, as well as digital controllers and supporting systems for active controls testing. These capabilities make the TDT the best-suited facility in the world for flutter testing large, full-span, aeroelastically-scaled models at transonic speeds.

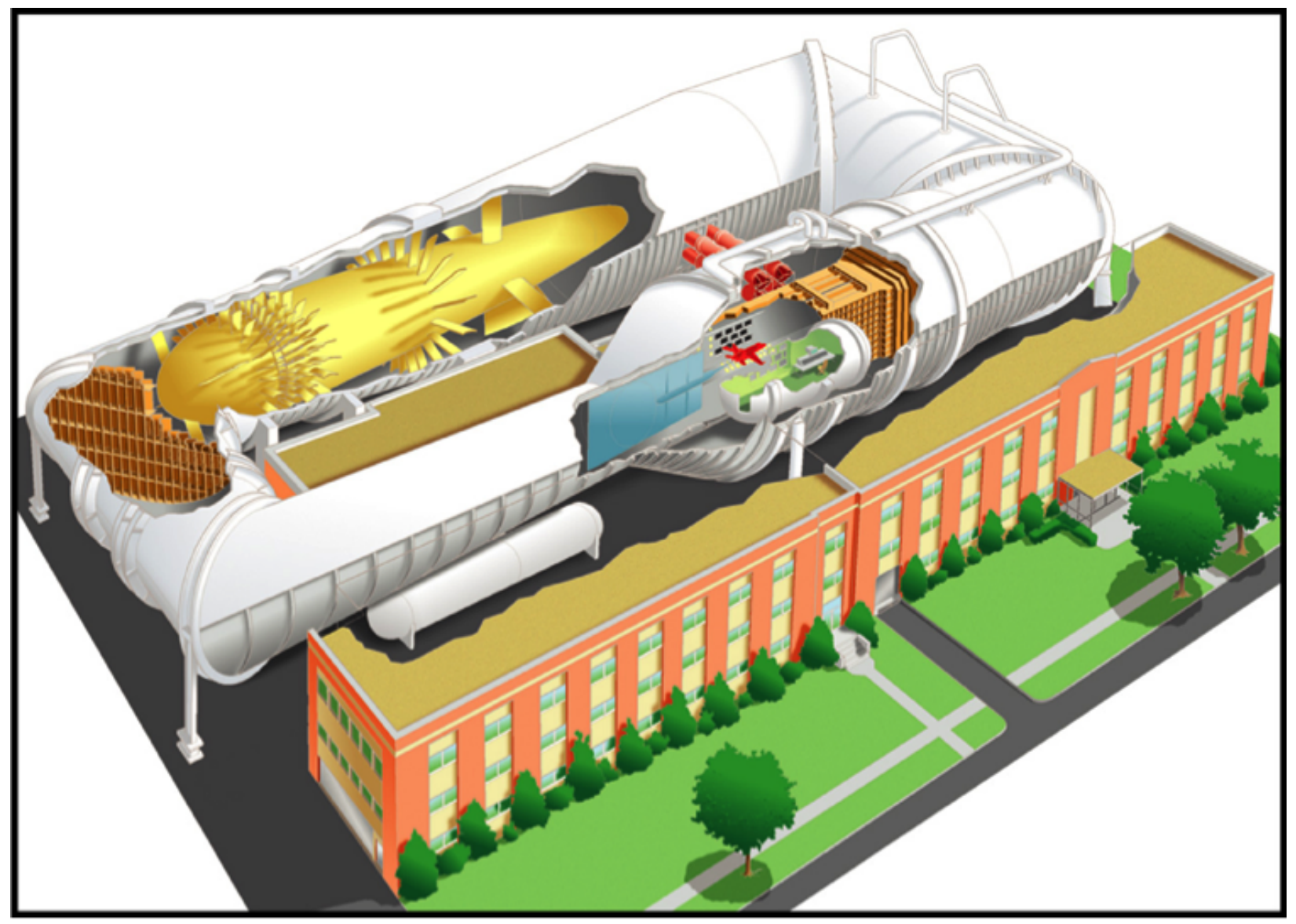

Figure 8: The Transonic Dynamics Tunnel (TDT).

\section{Computational Models}

The computational suite developed for this new wind-tunnel model includes a finite element model (FEM) and CFD grids. The FEM was initially developed at KTH, but it is continuously being updated as the windtunnel model hardware is fabricated and tested. To date, the FEM has been used to compute preliminary loads on the main components.

\section{Validation of the wing stiffness model}

As mentioned previously, the wing design is based on a sandwich structural concept featuring fiberglass reinforced epoxy wing skins over a foam core. There is also an internal fiberglass epoxy support frame for 
localized forces at wing pylon stations and wing root attachments. The internal frame is localized in the mid plane of the wing to minimize the contribution to overall wing stiffness while ensuring significant strength for high local forces when heavy external stores are attached to the wing.

The finite element model of the aircraft, shown in Figure 9, was developed for standard linear-elastic finite element analysis using NASTRAN [23].

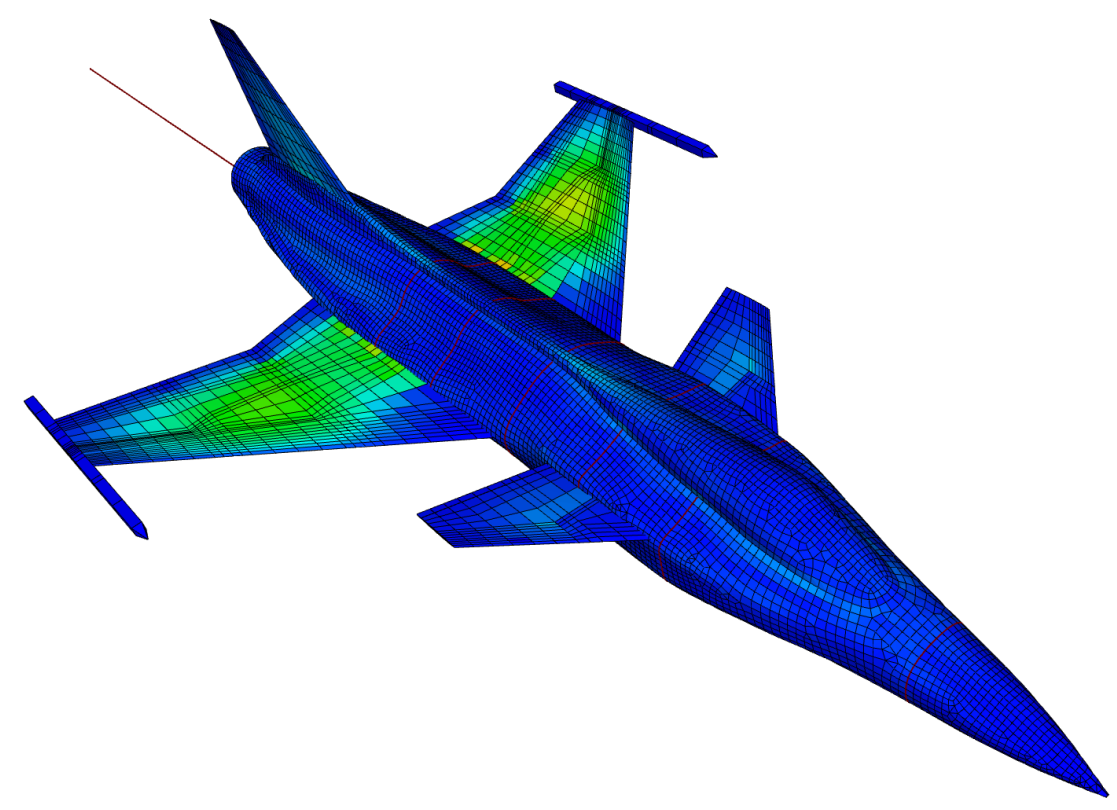

Figure 9: Finite element model of the aircraft on sting mount.

The core material is modeled using CHEXA solid elements while wing skins and internal reinforcements are modeled using CQUAD4 shell elements. There are 15718 total grid points and 17913 total elements in the FE model. Material properties for the composite laminates where derived by coupon testing of component specimens in a static testing machine as described in Passalacqua [24]. The remaining material properties were obtained from supplier data sheets of the materials used.

In order to verify the accuracy of the wing finite element model, a full-scale stiffness test is performed using the setup shown in Figure 6. Optical markers are attached to the wing hard points as shown in the Figure. The marker positions are recorded by an optical measurement system [20] using four motion capture cameras. The system is able to resolve displacements less than $0.1 \mathrm{~mm}$.

The system records the position of each marker in three dimensions with a data rate up to $1 \mathrm{kHz}$. For a static test such as this, the data rate is reduced to $60 \mathrm{~Hz}$ to limit the size of the data files generated. The external loads are applied using dead weights in increments of approximately $2 \mathrm{~kg}$. The weights are removed using the same increment to record the displacements for a full load cycle. Each load level is maintained for approximately 20 seconds, and the average position of each marker is used for the displacement estimate. The load is applied to the same hard point locations as used to attach the optical markers. This way a full matrix of flexibilities is measured.

The results obtained for the right wing tip markers and the markers at the outer under wing pylon station are shown in Figure 10.

The experimental displacement measurements are shown as ' + ' markers and the finite element model displacement results as a solid line. The measured displacement versus load response is close to a linear function.

The flexibility matrices are defined to be $8 \times 8$ using the vertical displacement measurements of the two 


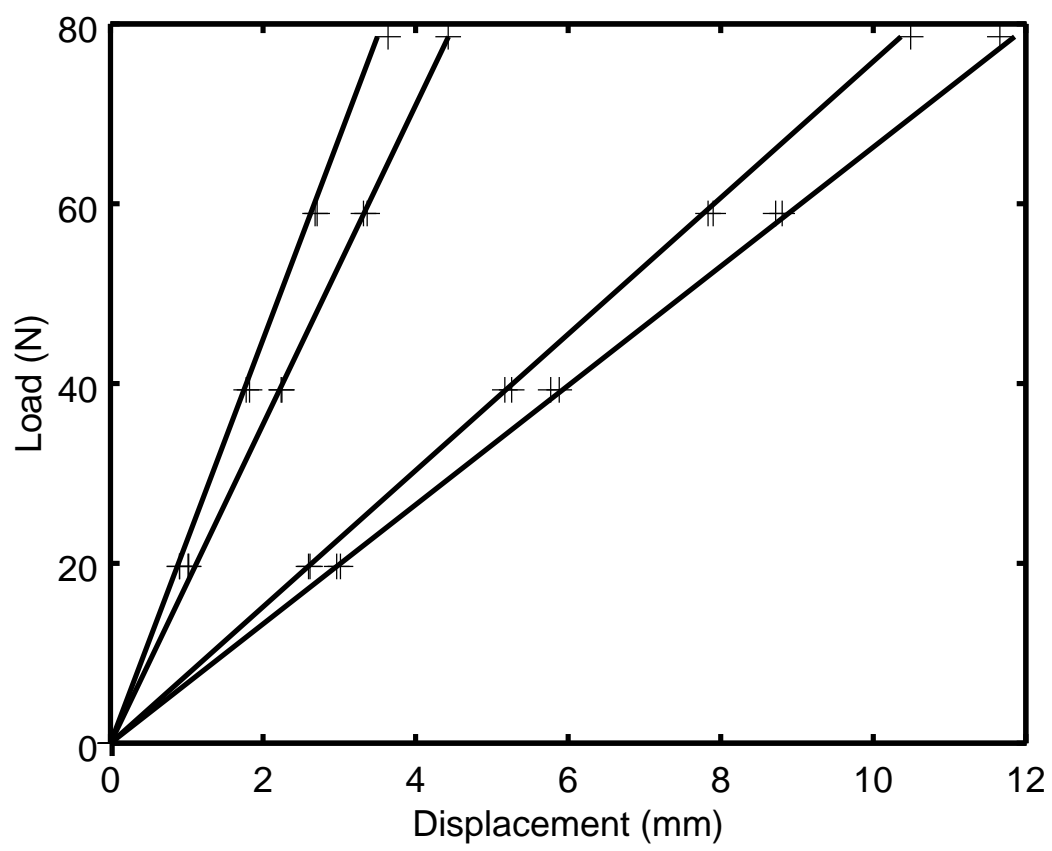

Figure 10: Outer wing marker displacement measurements and finite element model displacement results.

wing tip markers, the three markers at the outer under wing pylon station, and the three markers at the inner wing pylon station. The experimentally obtained flexibilities are obtained using a linear least-squares fit of the load versus deflection data as shown in Figure 10. The matrix of flexibilities from the finite element model $F_{\mathrm{FEM}}$ is derived by applying unit point loads to the nodes at wing hard points with one separate load case for each hard point.

The finite element model assumes that both wings have identical properties, but the flexibilities of both wings are experimentally recorded in the matrices $F_{\text {left }}$ for the left wing and $F_{\text {right }}$ for the right wing. To compare the flexibility of the two wings with the finite element model, non-dimensional matrix norm quantities are evaluated as shown in Table 1.

\begin{tabular}{lcc} 
Note & Matrix expression & Result \\
\hline Left symmetry & $\left\|F_{\text {left }}-F_{\text {left }}^{\mathrm{T}}\right\| /\left\|F_{\text {left }}\right\|$ & 0.014 \\
Right symmetry & $\left\|F_{\text {right }}-F_{\text {right }}^{\mathrm{T}}\right\| /\left\|F_{\text {right }}\right\|$ & 0.011 \\
Difference & $\left\|F_{\text {left }}-F_{\text {right }}\right\| /\left(\frac{1}{2}\left\|F_{\text {left }}+F_{\text {right }}\right\|\right)$ & 0.020 \\
Right vs FEM & $\left\|\frac{1}{2}\left(F_{\text {right }}+F_{\text {right }}^{\mathrm{T}}\right)-F_{\text {FEM }}\right\| /\left\|F_{\text {FEM }}\right\|$ & 0.039758 \\
Left vs FEM & $\left\|\frac{1}{2}\left(F_{\text {left }}+F_{\text {left }}^{\mathrm{T}}\right)-F_{\text {FEM }}\right\| /\left\|F_{\text {FEM }}\right\|$ & 0.030674 \\
Average vs FEM & $\left\|\frac{1}{4}\left(F_{\text {left }}+F_{\text {left }}^{\mathrm{T}}+F_{\text {right }}+F_{\text {right }}^{\mathrm{T}}\right)-F_{\text {FEM }}\right\| /\left\|F_{\text {FEM }}\right\|$ & 0.034568
\end{tabular}

Table 1: Comparison of flexibility matrices.

There is a small difference between the two wings, and the experimentally obtained matrices are not quite symmetric. The overall difference in flexibility between the finite element model and the experimental results is about $3-4 \%$. Tuning of material properties may reduce the difference between experimental results and the finite element model. 


\section{A. CFD Grids and Analyses}

Unstructured CFD grids have been generated at KTH for use with Reynolds-Averaged Navier-Stokes (RANS) codes. These grids include the wind-tunnel model configuration with and without the canards in order to determine the effect of the canards in preliminary analyses. Inviscid, steady, rigid solutions have been computed at several test conditions in order to determine the aerodynamic loads at those conditions. Presented in Figure 11 is a sample image of the grid and pressure distributions for the wind-tunnel model configuration with canards.

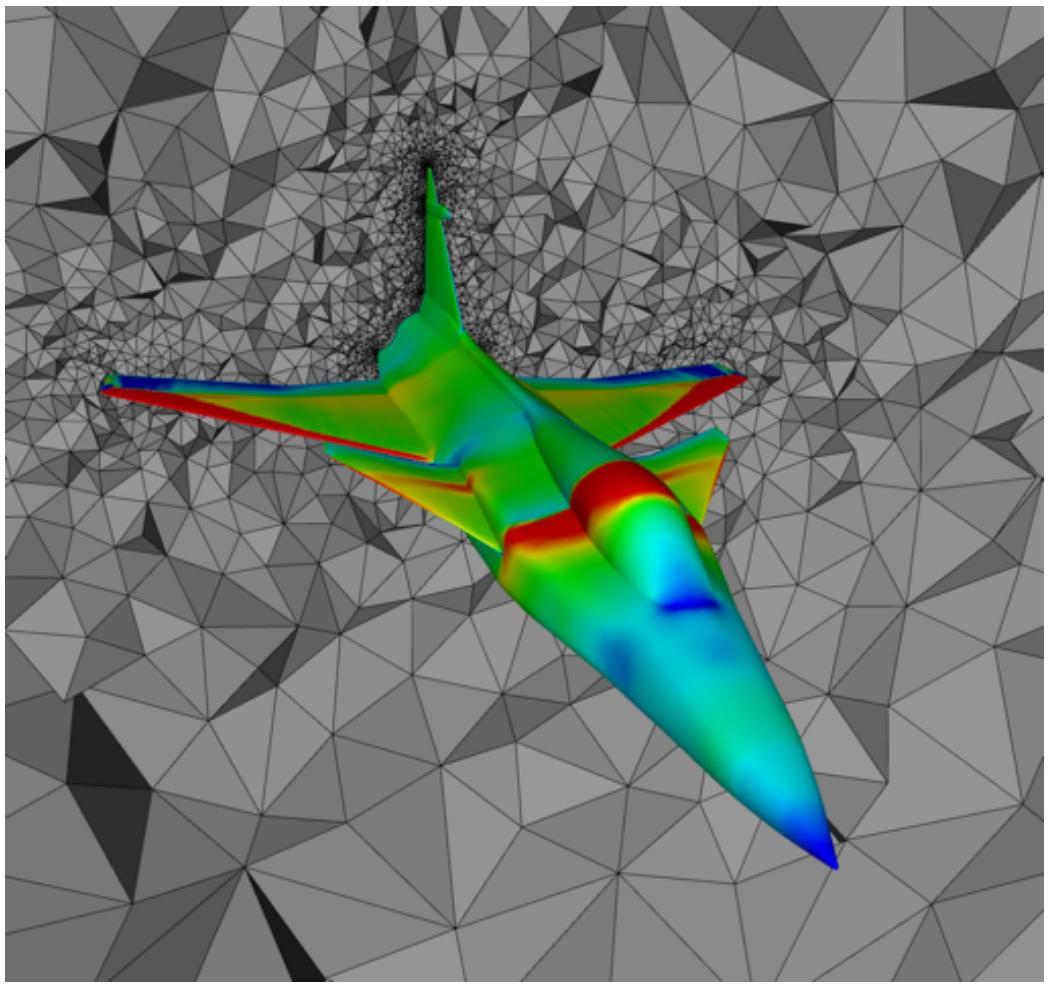

Figure 11: The CFD mesh of the wind-tunnel model with canards and steady pressures of the KTH-NASA aeroelastic wind-tunnel model at $\mathrm{M}=0.87, \mathrm{AoA}=7$ degrees.

Using the unstructured grid generated at KTH, NASA has also generated inviscid, steady, rigid solutions at $\mathrm{M}=0.9$ and 0 degrees angle of attack for the configuration without canards. Presented in Figure 12 is an image of the pressure distributions at this condition using the NASA-Langley-developed FUN3D RANS CFD code. Presented in Figure 13 are the Mach number distributions at $\mathrm{M}=0.9$ and $\mathrm{AoA}=0$ degrees using the FUN3D CFD code.

\section{Concluding Remarks}

The KTH-NASA collaboration has only recently begun. However, a significant amount of work has already been performed, and continues to be performed, by the KTH team. This includes design, fabrication, and testing of structural components and instrumentation. The NASA team is currently performing additional FEM and CFD analyses in support of the test and has begun preparation for the test itself which is planned for May 2016.

\section{References}

${ }^{1}$ Patil, M., "Limit-Cycle Oscillations of Aircraft Caused by Flutter-Induced Drag," AIAA Journal of Aircraft, Vol. 41, May 2004, pp. 571-576.

${ }^{2}$ Gopinath, A., Beran, P., and Jameson, A., "Comparative Analysis of Computational Methods for Limit-Cycle Oscilla- 


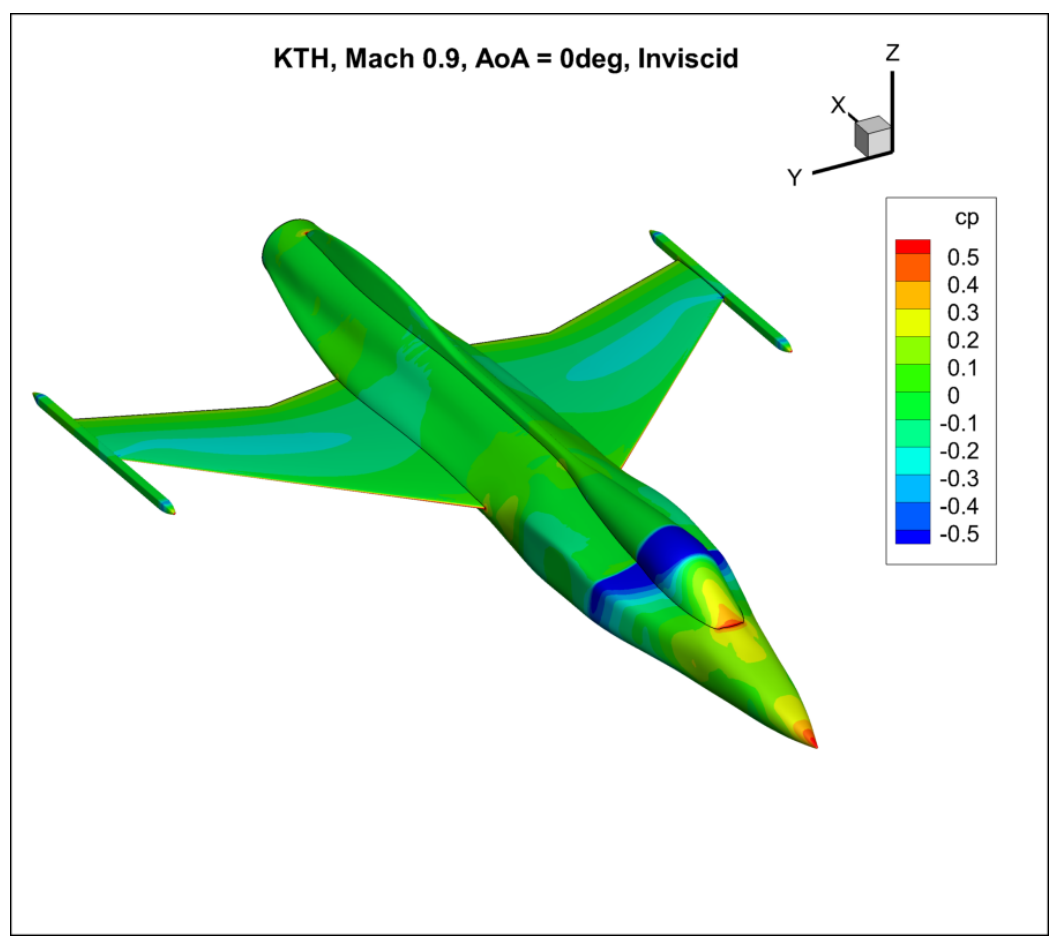

Figure 12: Pressure distributions at $\mathrm{M}=0.9, \mathrm{AoA}=0$ deg using FUN3D code.

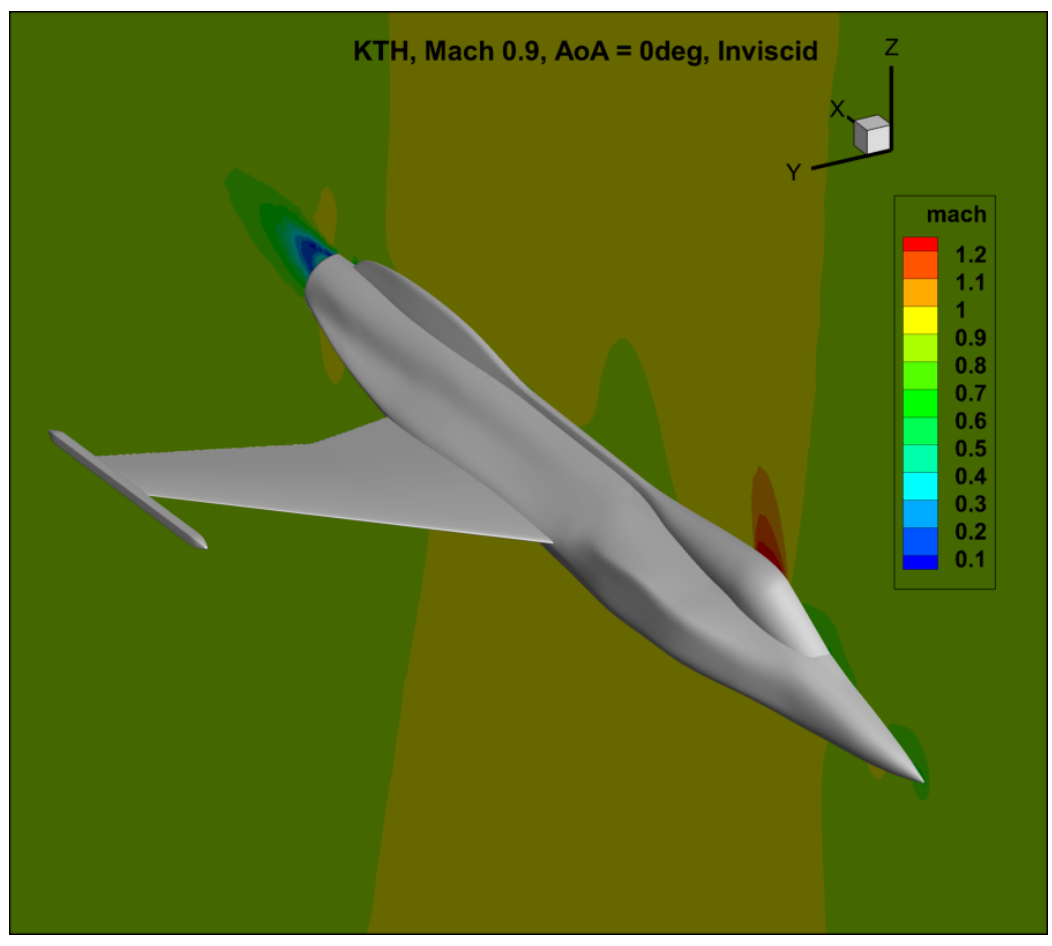

Figure 13: Mach number distributions at $\mathrm{M}=0.9, \mathrm{AoA}=0$ deg using FUN3D code. 
tions," 47th AIAA/ASME/ASCE/AHS/ASC Structures, Structural Dynamics, and Materials Conference, April 2006.

${ }^{3}$ Gordnier, R. E., "Computation of Limit-Cycle Oscillations of a Delta Wing," AIAA Journal of Aircraft, Vol. 40, Nov. 2003, pp. 1206-1208.

${ }^{4}$ Denegri, C. M., Dubben, J. A., and Maxwell, D. L., "In-Flight Wing Deformation Characteristics During Limit Cycle Oscillations," AIAA Journal of Aircraft, Vol. 42, March 2005, pp. 500-508.

${ }^{5}$ Padmanabhan, M. A., Pasiliao, C. L., and Dowell, E. H., "Simulation of Aeroelastic Limit-Cycle Oscillations of Aircraft Wings with Stores," AIAA Journal, Vol. 52, Oct. 2014, pp. 2291-2299.

${ }^{6}$ Bunton, R. W. and Denegri, C. M., "Limit Cycle Oscillation Characteristics of Fighter Aircraft," AIAA Journal of Aircraft, Vol. 37, Sept. 2000, pp. 916-918.

${ }^{7}$ Yao, W. and Marques, S., "Prediction of Transonic Limit-Cycle Oscillations Using an Aeroelastic Harmonic Balance Method," AIAA Journal, April 2015.

${ }^{8}$ Sheta, E. F., Harrand, V. J., Thompson, D. E., and Strganac, T. W., "Computational and Experimental Investigation of Limit Cycle Oscillations of Nonlinear Aeroelastic Systems," AIAA Journal of Aircraft, Vol. 39, Jan. 2002, pp. 133-141.

${ }^{9}$ Bendiksen, O., "Transonic Limit Cycle Flutter/LCO," 45th AIAA/ASME/ASCE/AHS/ASC Structures, Structural Dynamics and Materials Conference, April 2004.

${ }^{10}$ Silva, W., Brenner, M., Cooper, J., Denegri, C., Dunn, S., Huttsell, L., Kaynes, I., Lind, R., Poirel, D., and Yurkovich, R., "Advanced Flutter and LCO Prediction Tools for Flight Test Risk and Cost Reduction - An International Collaborative Program for T\&E Support," 2005 U.S. Air Force TEE Days, Dec. 2005.

${ }^{11}$ Chabalko, C., Hajj, M., Mook, D., and Silva, W., "Characterization of the LCO Response Behaviors of the NATA model," 47th AIAA/ASME/ASCE/AHS/ASC Structures, Structural Dynamics, and Materials Conference, April 2006.

${ }^{12}$ Silva, W. and Dunn, S., "Higher-Order Spectral Analysis of F-18 Flight Flutter Data," 46th AIAA/ASME/ASCE/AHS/ASC Structures, Structural Dynamics and Materials Conference, April 2005.

${ }^{13}$ Tomac, M. and Eller, D., "From geometry to CFD grid: An automated approach for conceptual design," Progress in Aerospace Sciences, April 2011.

${ }^{14}$ Eller, D., "An efficient boundary element method for unsteady low-speed aerodynamics in the time domain," TRITA/AVE 2005:40, Stockholm, Sweden, April 2005.

${ }^{15}$ Modin, K. E. and Clareus, U., "Aerodynamic design evolution of the SAAB JAS 39 Gripen aircraft," AIAA/AHS/ASEE Aircraft design systems and operations meeting, April 1991. 2002.

${ }^{16}$ Eliasson, P., "EDGE, a Navier-Stokes solver for unstructured grids," Finite Volumes for Complex Applications III, April

${ }^{17}$ Stenfelt, G., "Aerodynamics and lateral control of tailless aircraft," PhD Thesis, March 2012.

${ }^{18}$ Jansson, N. and Stenfelt, G., "Steady and unsteady pressure measurements on a swept-wing aircraft," Technical Report TRITA/AVE 2011:91, April 2011.

${ }^{19}$ Huebner, A. R., Bergmann, A., and Loeser, T., "Experimental and numerical investigations of unsteady force and pressure distributions of moving transport aircraft configurations," 47th AIAA Aerospace Sciences Meeting Including The New Horizons Forum and Aerospace Exposition, No. AIAA paper 2009-91, Jan. 2009.

${ }^{20}$ Qualisys AB, Oqus - Qualisys motion capture camera with high-speed video, Product Information 100, 300 and 500 series, 2011.

${ }^{21}$ Corliss, J. M. and Cole, S. R., "Heavy Gas Conversion of the NASA Langley Transonic Dynamics Tunnel," Proceedings of the 20th Advanced Measurements and Ground Testing Technology Conference, No. 98-2710, Albuquerque, NM, June 1998.

${ }^{22}$ Cole, S. R. and Rivera Jr, J. A., "The New Heavy Gas Testing Capability in the NASA Langley Transonic Dynamics Tunnel," Royal Aeronautical Society Wind Tunnels and Wind Tunnel Test Techniques Forum, No. No. 4, Cambridge, UK, April 1997.

${ }^{23}$ Siemens Product Lifecycle Management Software Inc., NX Nastran 8 Quick Reference Guide, 2011.

${ }^{24}$ Passalacqua, F., "Characterization of glass fiber reinforced material through off-axis tensile tests," Tech. rep., Department of Aeronautical and Vehicle Engineering, 2014. 